Aus der Neuropathologischen Abteilung (Prof. Dr. K. Scharenberg) der Psychiatrischen Klinik (Prof. Dr. R. W. Waggoner) des Universitätskrankenhauses Ann Arbor, Michigan, USA

\title{
Die Nerven und das Bindegewebe der Pia des Menschen im mikrophotographischen Bild*
}

Von

\section{K. Scharenberg:*}

Mit 21 Textabbildungen

Die Kenntnisse von der histologischen Struktur der Pia des Menschen sind trotz der Bemühungen zahlreicher Autoren sehr begrenzt. Zwar ist das Nervensystem der weichen Himhaut untersucht worden - hauptsächlich von $P h$. Stöhr jr. ${ }^{1}$ - und kann als teilweise bekannt gelten. Jedoch müssen die Ergebnisse aller bisherigen Untersuchungen nur als Fragmente gewertet werden; das Gesamtbild fehlt noch. Noch weniger bekannt sind die komplizierten Bindegewebsstrukturen der Pia. Bis auf eine Arbeit von Schultz und Knibbe ${ }^{2}$ und ältere Arbeiten von Key und Retzius ${ }^{3}$ finden sich im Schrifttum keinerlei Angaben darüber.

Die älteren technischen Verfahren - inklusive der älteren Silbermethoden wie der von Schultze, Gross, Bielschowsky und der reduzierten Silbermethode von Cajal - sind für die Imprägnierung von Nerven unzuverlässig und nicht selektiv; für die Darstellung des Bindegewebes sind sie wertlos. Daher können auch die mit diesen Methoden erhobenen Befunde photographisch kaum wiedergegeben werden. Die Einführung und Entwicklung der Silberkarbonattechnik durch del Rio Hortega erlauben eine getrennte Imprägnierung der Nerven und des Bindegewebes und ermöglichen eine klare mikrophotographische Wiedergabe der Befunde. Letzteres vor allen Dingen ist die Aufgabe dieser Arbeit.

\section{Material und Methodik}

Für die Untersuchungen wurden zirka $1 \times 2 \mathrm{~cm}$ große normale Piastücke benutzt. Diese wurden als Plättchen nach der Methode von Schultz und Knibbe vorsichtig vom frischen Gehirn abgelöst und in Bromformalin fixiert. Für die Imprägnierung der Nerven wurde die unreduzierte

* In memoriam Prof. Dr. Albert Kuntz, dem hervorragenden Forscher des vegetativen Nervensystems.

** Mit Unterstützung des Grant No. B-418-C 3, des U. S. Department of Public Health, Education, and Welfare. 
und die panoptische Variante von del Rio Hortega, für die Darstellung des Bindegewebes die dreifache Variante nach del Rio Hortega-Scharenberg ${ }^{4}$, angewandt. Mit einigen Einschränkungen handelt es sich also um Gesamtpräparate. Es ist aber zu bedenken, daß bei der Ablösung der Pia die Strukturen, welche die weiche Hirnhaut mit der marginalen Hirnschicht verbinden, zerstört werden und daß nicht selten einzelne Schichten der Pia mit abgerissen werden. Das letztere ist aber ein Vorteil, der es ermöglicht, verschiedene Schichten einzeln zu imprägnieren. Mikrotomschnitte wurden nicht angefertigt. Alle Abbildungen sind unretouchierte Mikrophotographien.

\section{Histologische Befunde und Erörterung}

Es ist bekannt, daß die Nerven der Pia teils von der Dura kommen, von deren Innenfläche sie brückenartig zur Pia herüberziehen (vgl. Kautzky und Wolter ${ }^{5}$ ) und teils mit den Arterien zur Pia gelangen (sogenannter

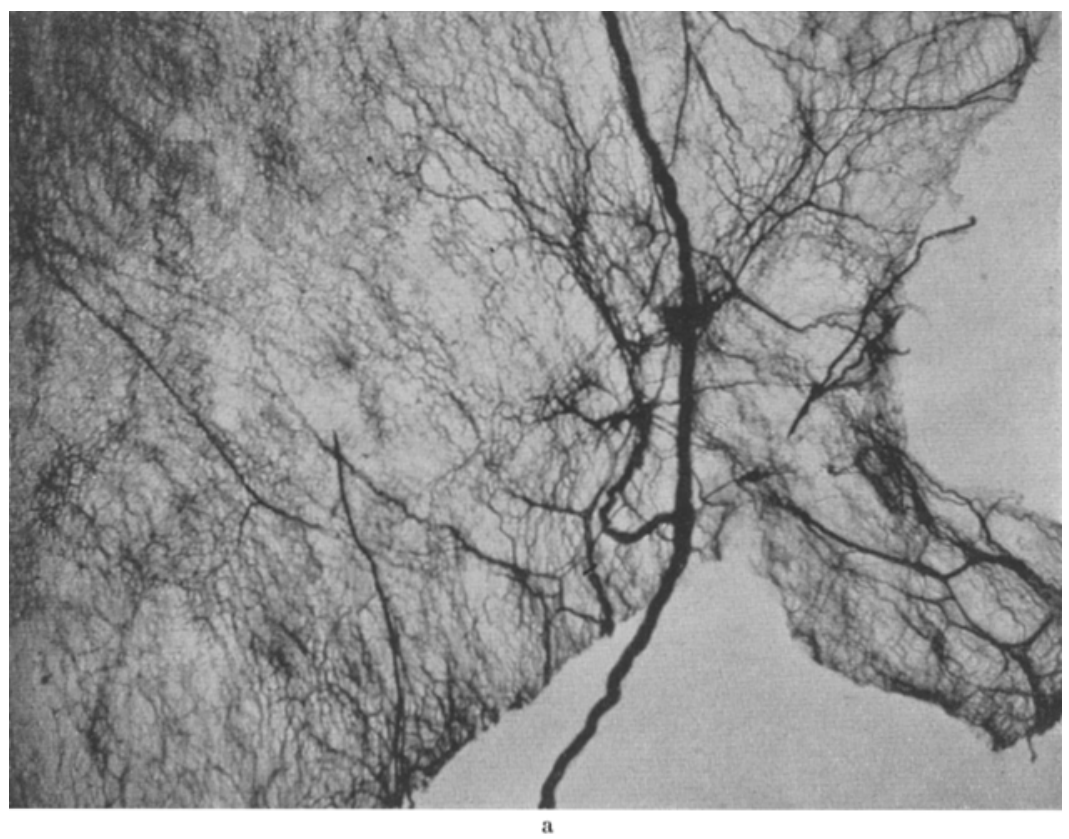

Abb. I. Ein Zweig eines spinalen Nerven (a) tritt in die Pia ein und gibt zahlreiche Aste an das Grundnetz $a b$; dieses ist außerordentlich stark entwickelt. - Silberkarbonat, unreduzierte Variante; $\times 20$.

sympathischer Gefäßplexus). Die Nerven der Pia können in 2 Systeme eingeteilt werden. Das sind: 1. direkt zu den Gefäßen verlaufende Nerven und 2. Nerven, welche in der Hauptsache das nervöse Grundnetz bilden. Beide Systeme sind durch zahlreiche Anastomosen miteinander verbunden.

1. Die Nerven der Gefäße: Der Nerv , $\mathrm{a}$ " in Abb. 1 zieht nach seinem Eintritt in die Pia zu einem größeren Gefäß und beteiligt sich an der Bil- 


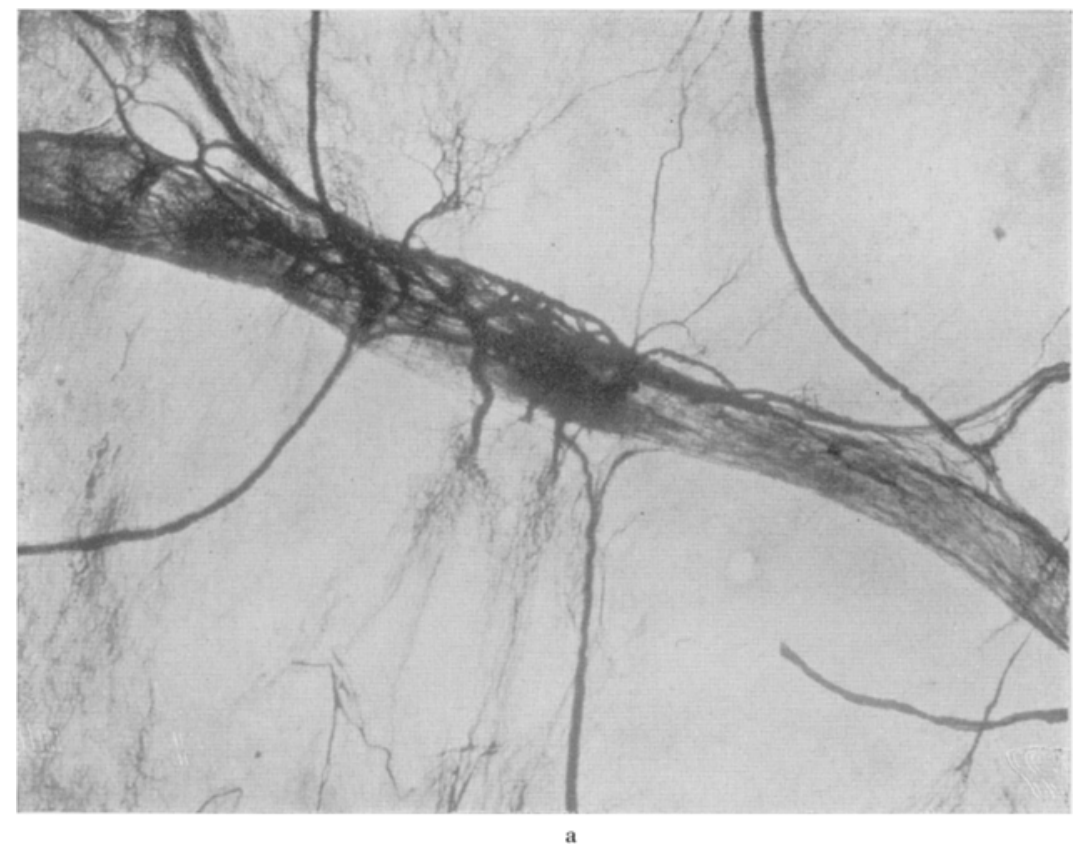

Abb. 2. Der Nerv "a ${ }^{\text {“ }}$ erreicht das Gefäß und beteiligt sich mit mehreren anderen Nerven an der Bildung eines perivaskulären Plexus, - Silberkarbonat, unreduzierte Variante; $\times 40$.

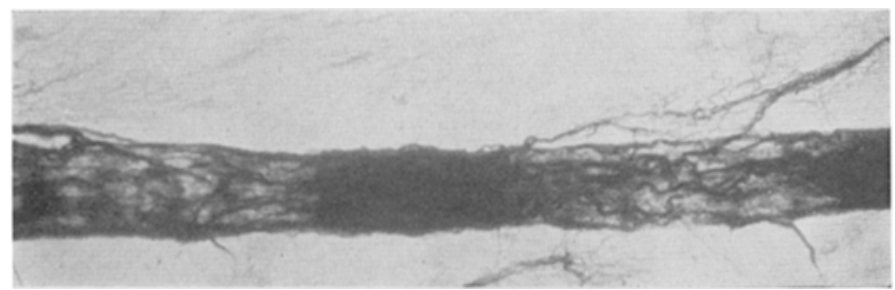

Abb. 3. Zahlreiche perivaskuläre Nerven bilden einen dichten kontinuierlichen Plexus. - Silberkarbonat, unreduzierte Variante; $\times 40$.

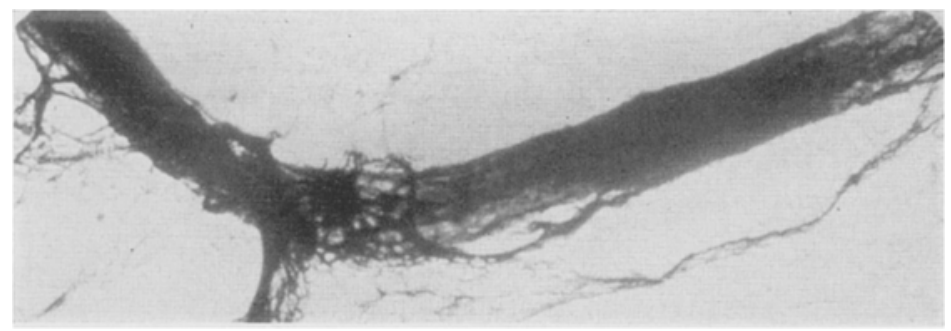

Abb. 4. Ein grobmaschiger, perivaskulärer Plexus vom Typ einer ,fenestrierten Membran“. Silberkarbonat, unreduzierte Variante; $\times \mathbf{4 0}$. 
dung eines perivaskulären Plexus (Abb. 2). Der mittlere Abschnitt dieses Plexus wird von Nerven stärkeren Kalibers gebildet, die sich allmählich in ein immer feineres Netzwerk aufsplittern (Abb, 2 und 3). Etwas abweichend ist die Struktur des perivaskulären Plexus in Abb. 4, der mehr einer fenestrierten nervösen Membran gleicht. Es sind immer zahlreiche Nervenstämme an der Bildung der perivaskulären Plexus beteiligt (Abb. 2).

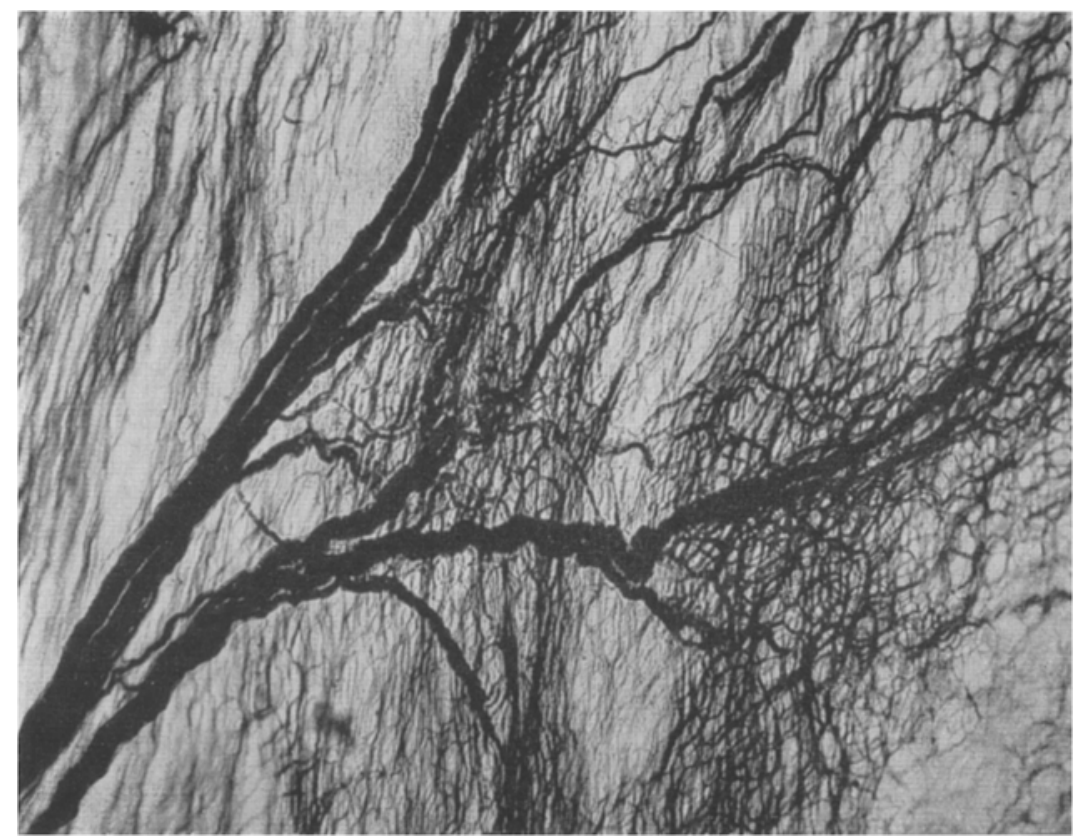

Abb. 5. Zwei starke Nervenbündel, welche nach dem Eintritt in die Pia im Grundnetz aufgehen. - Silberkarbonat, unreduzierte Variante; $\times 40$.

2. Das nervöse Grundnetz wird aus mehreren Komponenten aufgebaut: a) aus Nerven, welche nach dem Eintritt in die Pia und einem meistens kurzen Verlauf zahlreiche Äste abgeben und in keine unmittelbare Verbindung zu den Blutgefäßen treten (Abb. 5); b) aus Nervenästen, welche von einem größeren Nervenstamm, der zu einem Gefäß läuft, abzweigen (Abb. 1 und 6); c) aus zahlreichen Nervenbündeln, die von Gefäßnerven stammen und dreieckige Plexus bilden, deren Ausläufer im Grundnetz aufgehen (Abb. 7). Mitunter verlassen solche Äste das Blutgefäß im rechten Winkel und breiten sich wurzelartig im Grundnetz aus (Abb. 8).

Wie innig die perivaskulären Plexus mit dem Grundnetz verbunden sind, wird durch Abb. 9 veranschaulicht. Darauf sieht man, wie zwei aus perivaskulären Nerven hervorgehende Abzweigungen in der Nähe des Gefäßes ein dichtes Flechtwerk bilden, das in das Grundnetz übergeht. d) Einen wichtigen Bestandteil des nervösen Grundnetzes bilden Nerven, welche gleich 


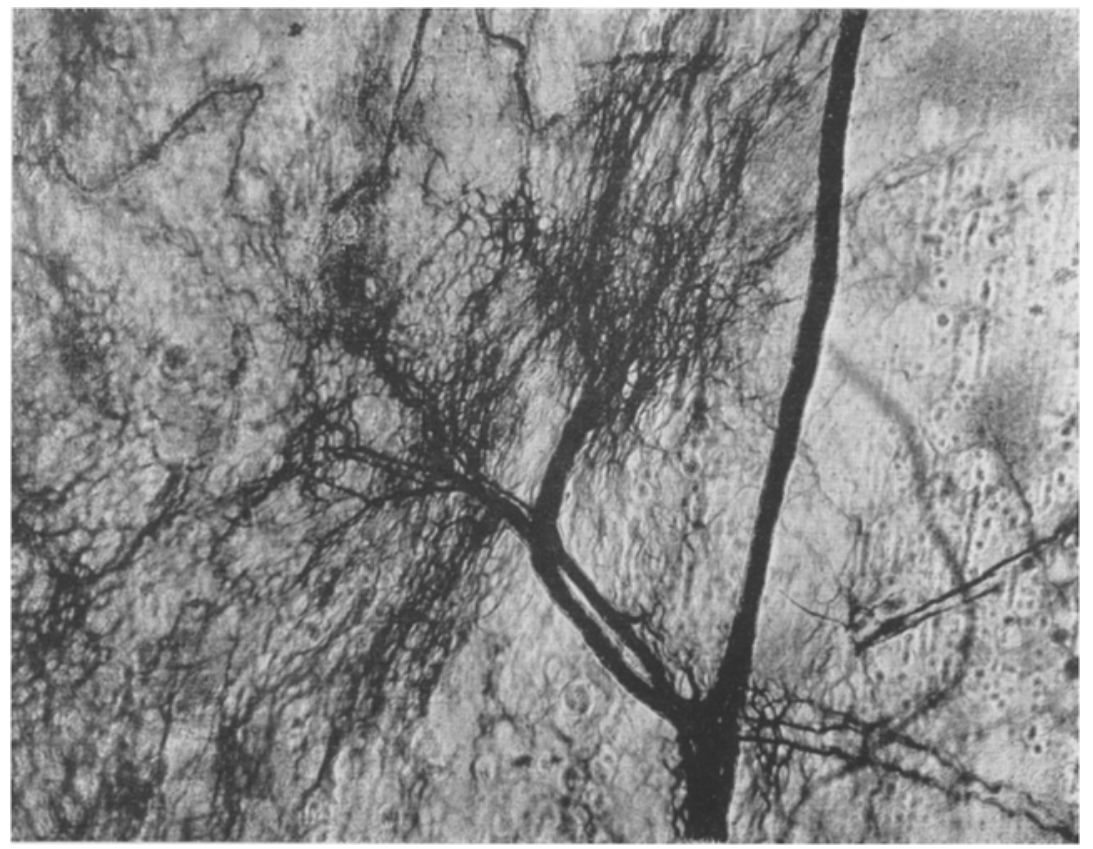

Abb. 6. Starke Äste eines zum Gefäß verlaufenden Nerven zweigen sich von demselben ab und verstärken das Grundnetz. - Silberkarbonat, unreduzierte Variante; $\times 60$.

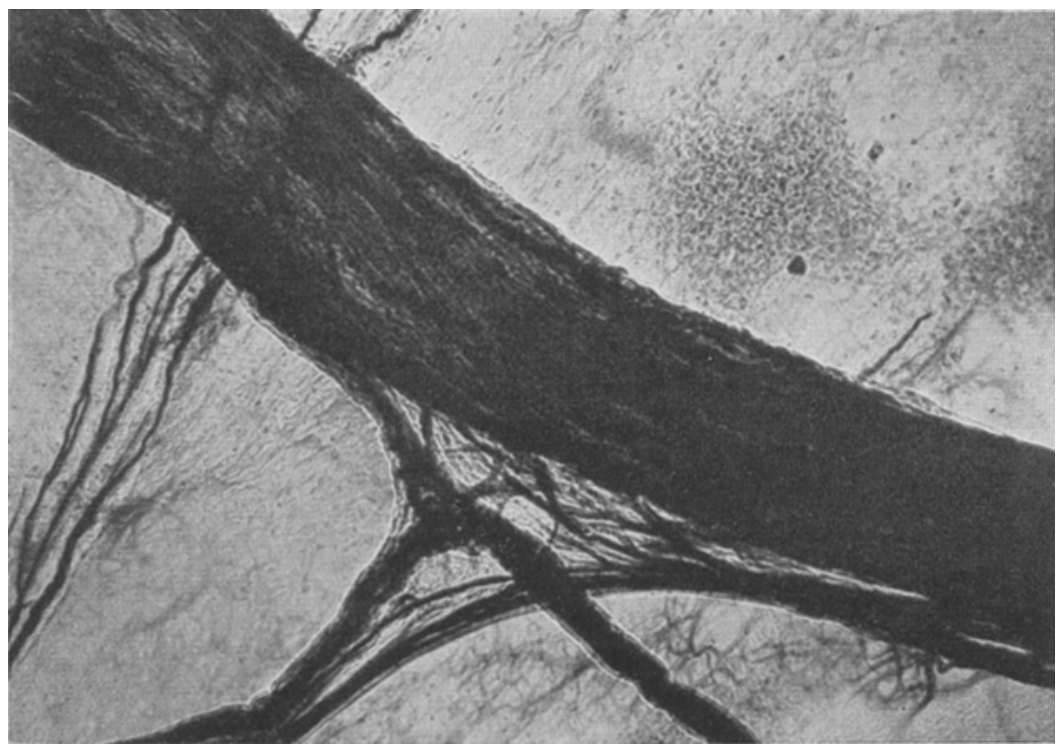

Abb.7. Zwei vom Gefäßplexus abzweigende Nerven bilden einen dreieckigen Plexus und gehen im Grundnetz auf. Links im Bild einer der zahlreichen Plexus des Grundnetzes; rechts eine Gruppe von Kernen der Arachnoidea. - Silberkarbonat, unreduzierte Variante; $X 40$. 
K. Scharenberg: Die Nerven und das Bindegewebe der Pia des Menschen 283

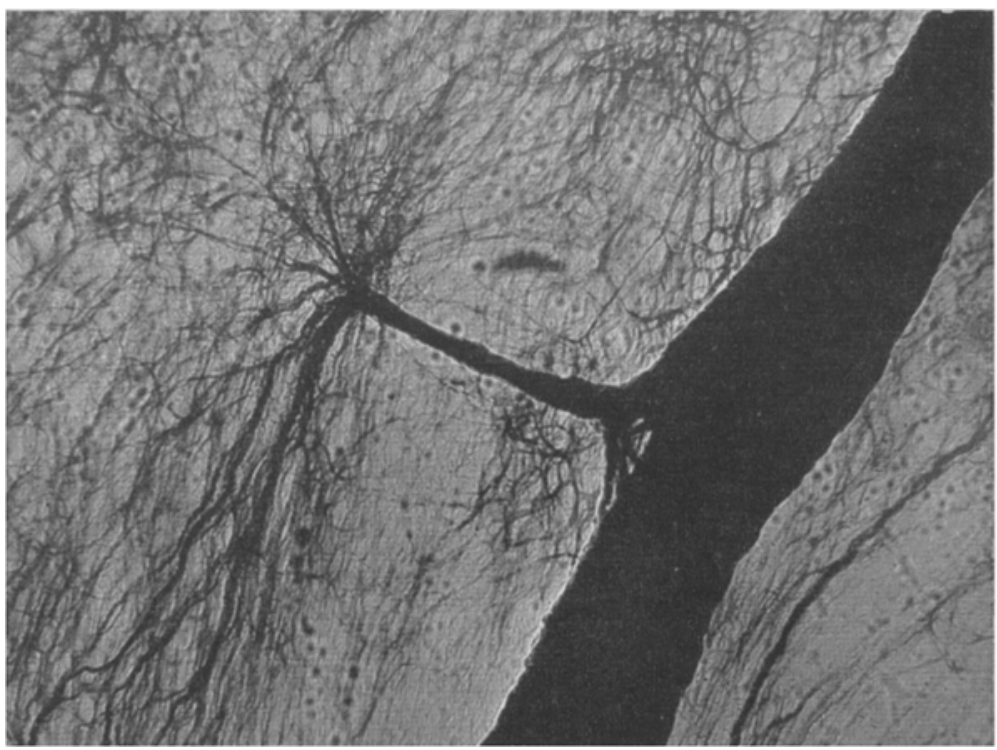

Abb. 8. Ein vom Gefäßplexus rechtwinkelig abgehender Nerv breitet sich wurzelartig im nervösen Grundnetz aus. - Silberkarbonat, unreduzierte Variante; $\dot{X} 60$.

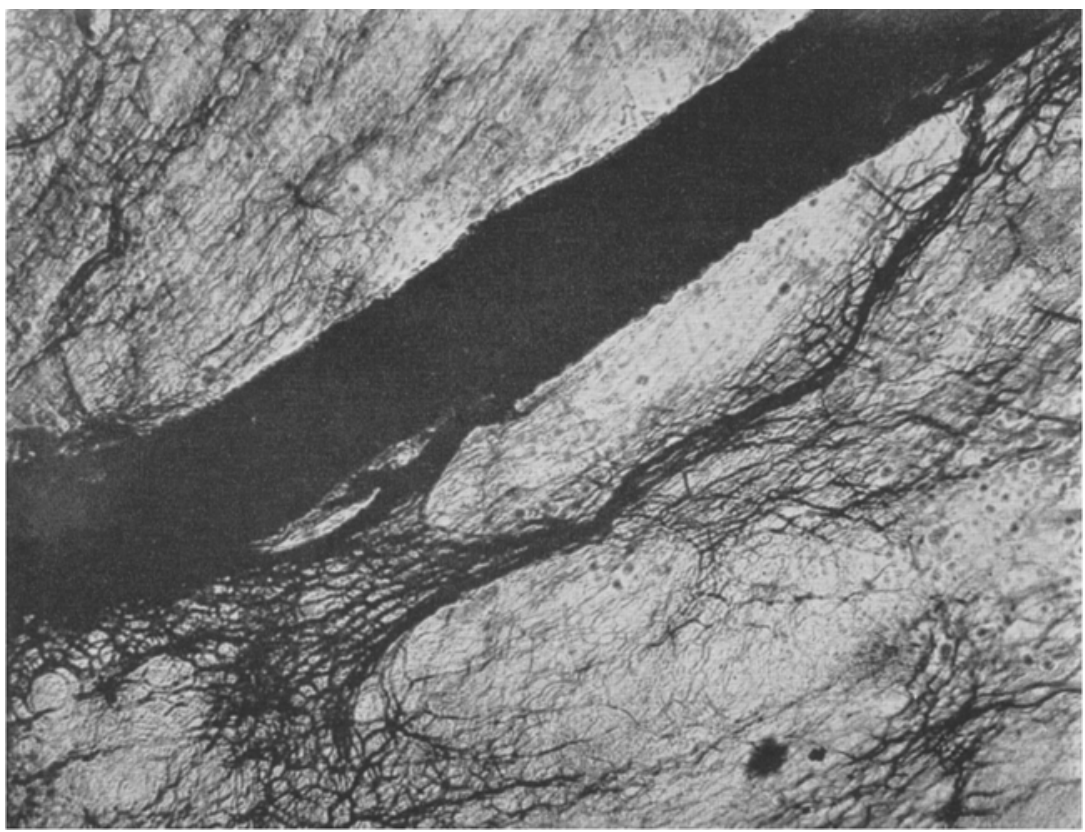

Abb. 9. Zwei rückläufige Nerven eines perivaskulären Plexus bilden ein dichtes Netz, welches im Grundnetz aufgeht. - Silberkarbonat, unreduzierte Variante; $\times 40$. 
nach ihrem Eintritt in die Pia grobmaschige Plexus aufbauen (Abb. 10); dieselben sind durch zahlreiche Kollateralen mit benachbarten Plexus verbunden (Abb. 11) und verlieren sich allmählich im Grundnetz (Abb. 12).

Das Grundnetz erscheint histologisch als eine unentwirrbare nervöse Struktur, welche ein in sich geschlossenes Ganzes darstellt und keinerlei besondere Endformationen erkennen läßt (Abb. 13).

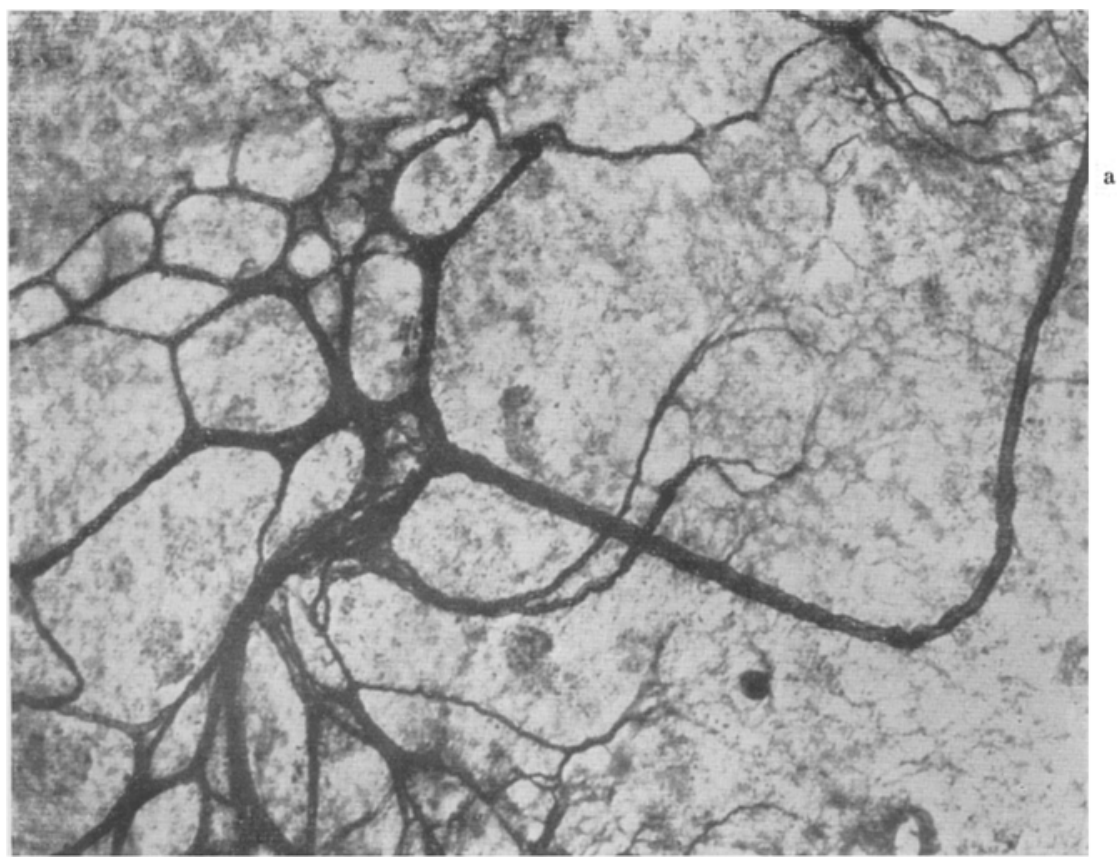

Abb. 10. Der Nerv , $\mathrm{a}^{a}$ bildet einen grobmaschigen stark verzweigten Plexus im nervösen Grundnetz. - Silberkarbonat, panoptische Variante, $\times 20$.

Nervenendapparate wurden nur an den Blutgefäßen nachgewiesen. Sie bestehen aus verzweigten Gebilden, die der Media aufliegen oder sich in unmittelbarer Nähe derselben befinden. Dieser Befund gleicht den von Boecke ${ }^{7}$ beschriebenen sympathischen Fasern der Kapillaren und der quergestreiften Muskeifasern (Abb. 14).

Das Bindegewebe der Pia ist nicht weniger kompliziert gebaut als die nervösen Strukturen. Es besteht genau wie die Nerven aus 2 verschieden gebauten Systemen: A. aus Strukturen der Adventitia, und B. aus dem Fibrillennetz, das die Gewebsmaschen ausfülltt.

A. Die Gefäße sind von einem Netzwerk von Fasern umgeben, in dem 4 Lagen unterschieden werden können. 1. die oberste Schicht der Adventitia, die als ein dichtes Netz meist parallel verlaufender starker Fasern am deutlichsten in den Präparaten erscheint, welche die Gesamttiefe der Pia enthalten (Abb. 15). Die 2. Bindegewebsschicht enthält große birnen- 


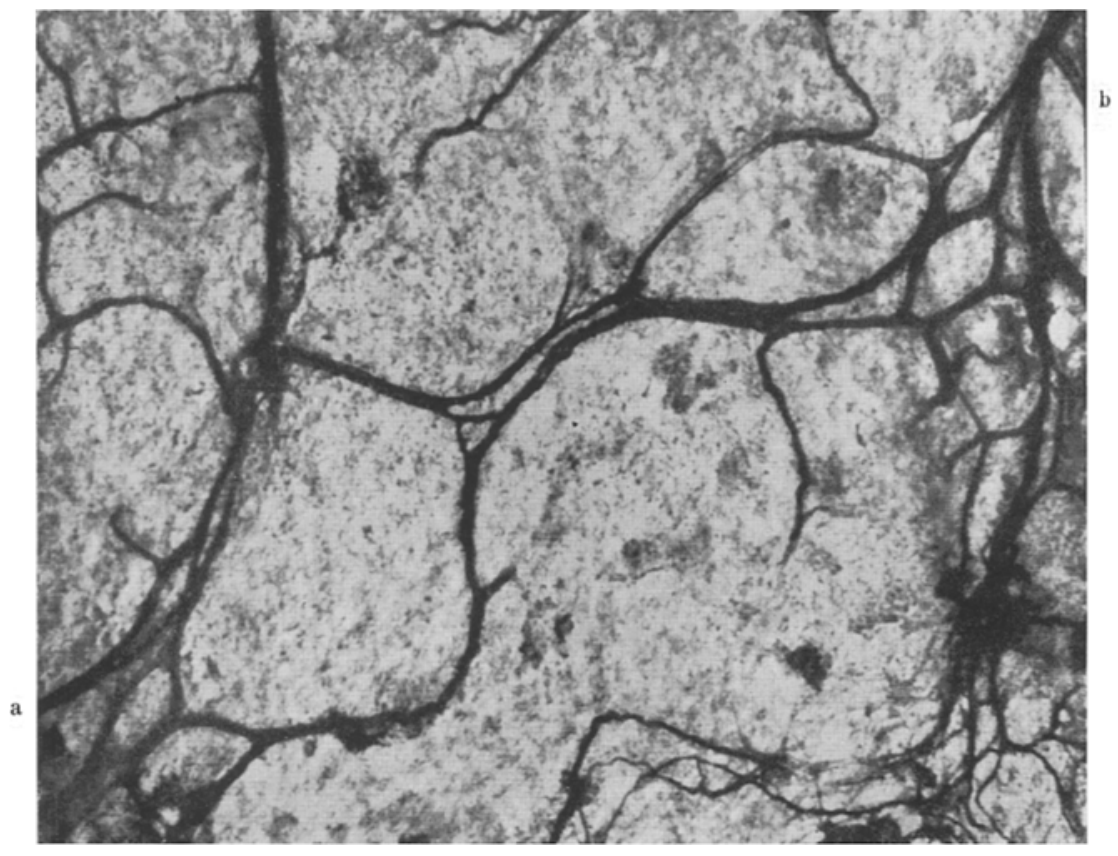

Abb. 11. Kollateralen des Plexus, , $\mathrm{a}^{: "}$ von Abb. 10 verlaufen zum Plexus , , ${ }^{\mathrm{c}}$. - Silberkarbonat, panopische Variante; $\times 40$.

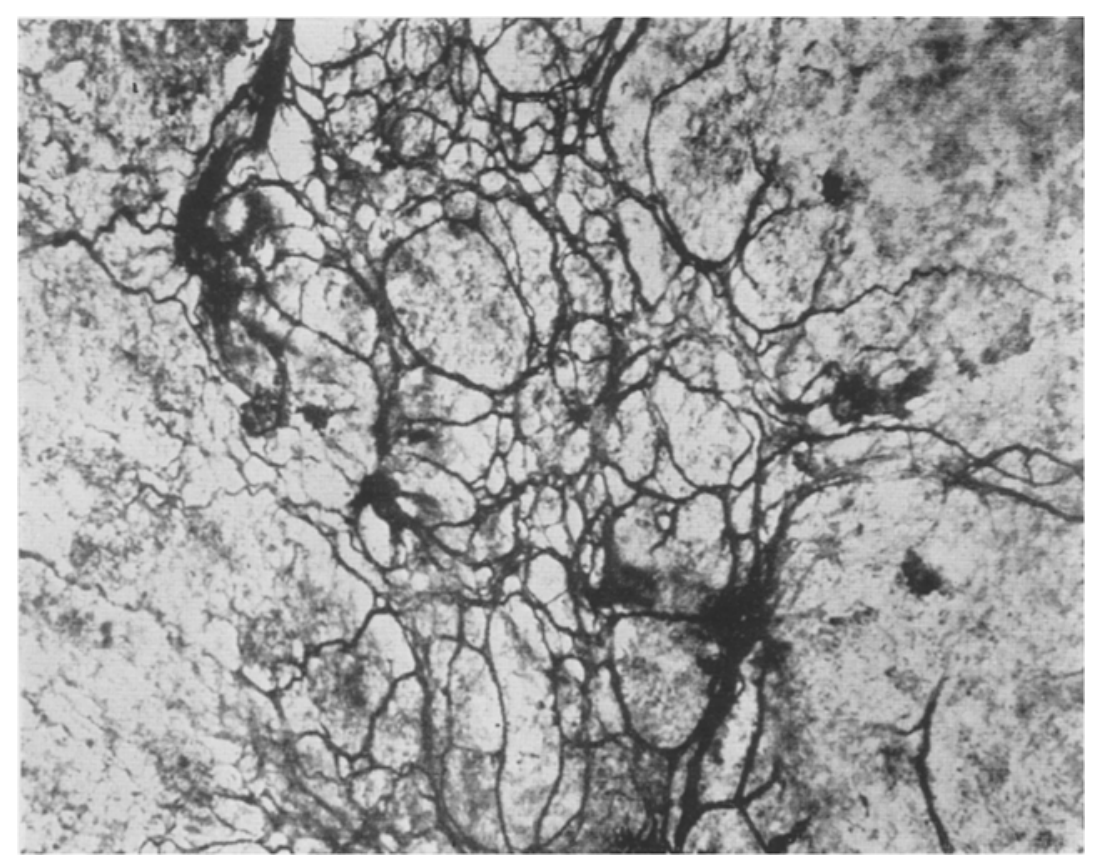

Abb. 12. Der Plexus von Abb. 10 geht allmählich in das Grundnetz über. - Silberkarbonat, panoptische Variante; $\times 40$. 


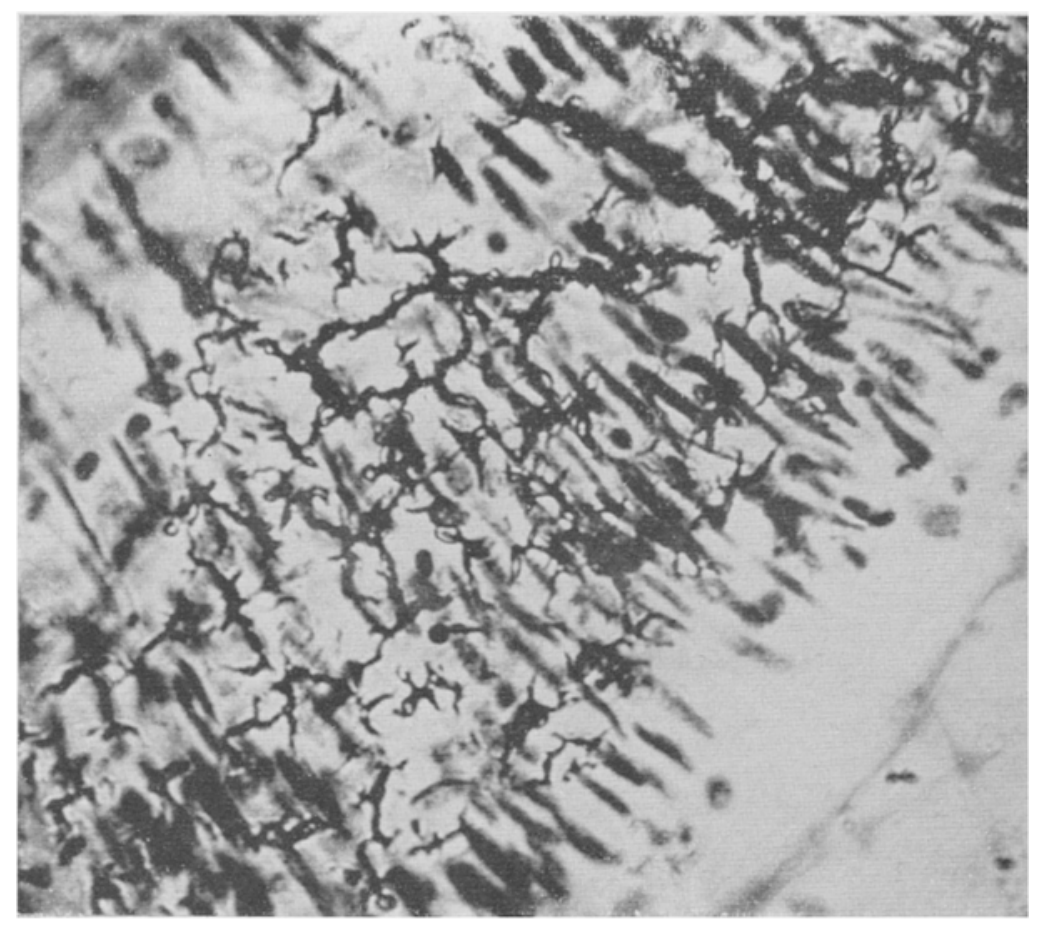

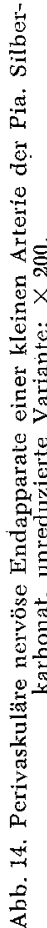
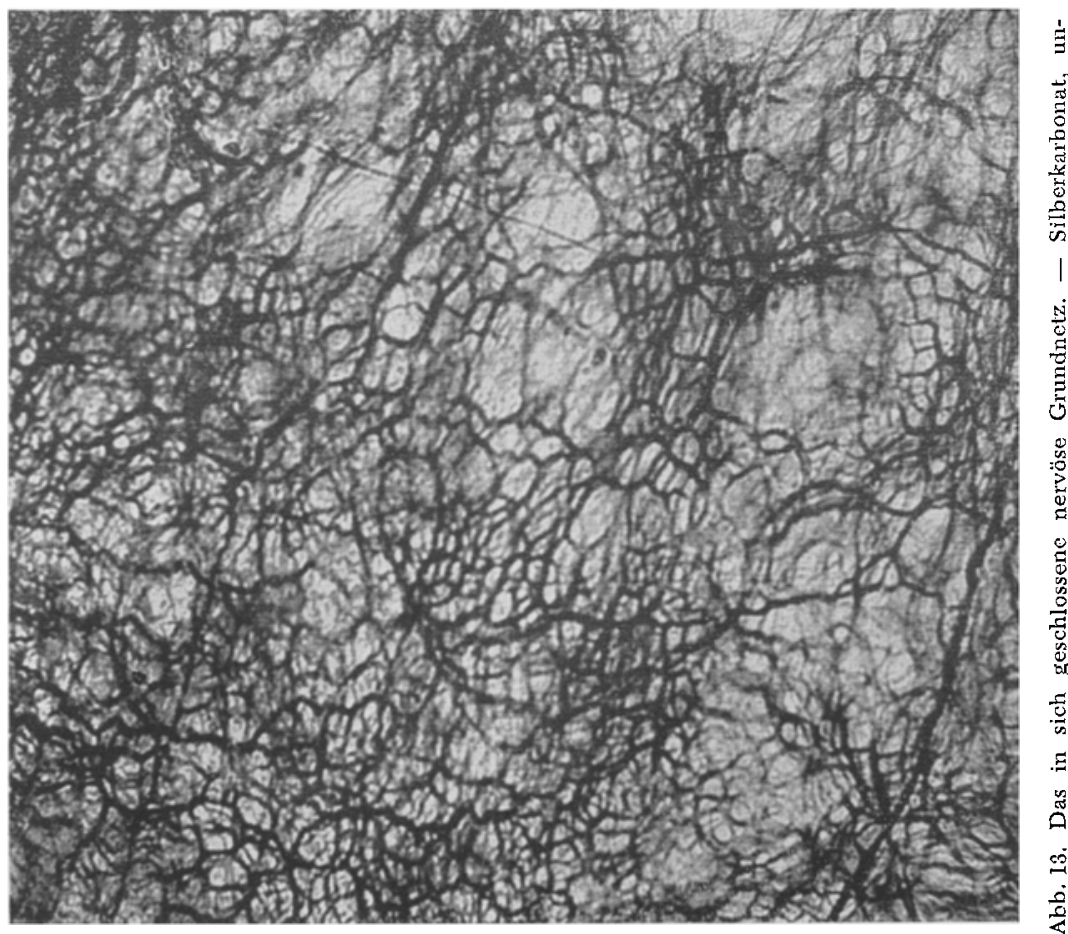


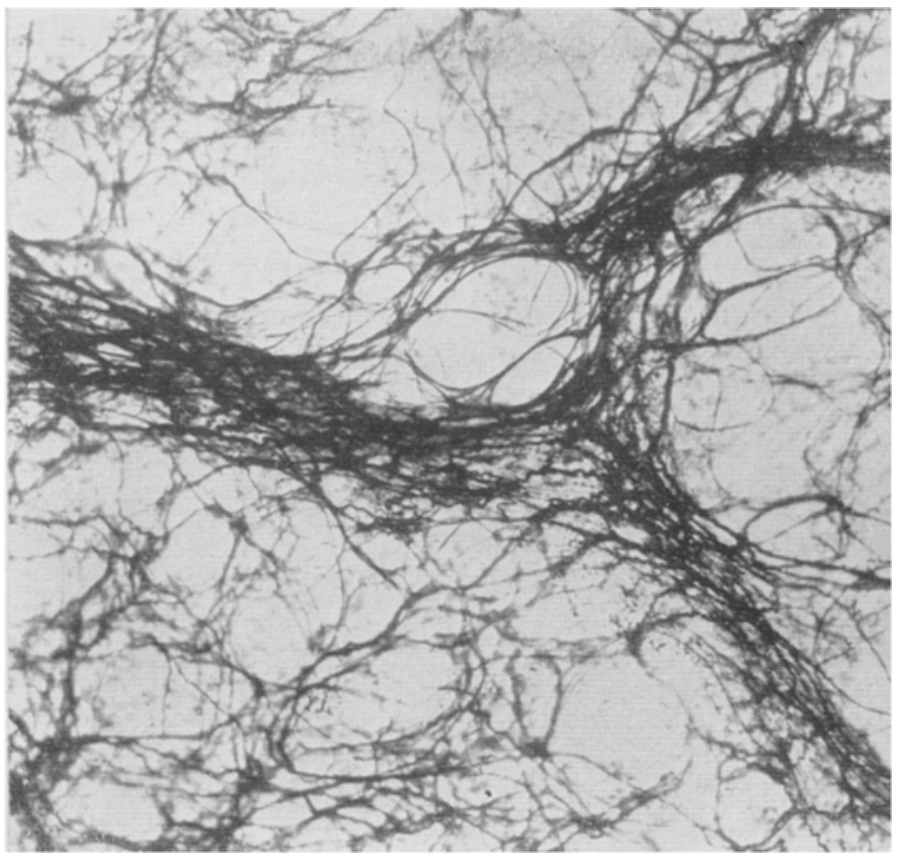

Abb. 15. Oberfächliche Schicht der Adventitia eines kleinen Gefäßes der Pia. - Silberkarbonat, Bindegewebsvariante nach Hortega-Scharenberg; $\times 40$.

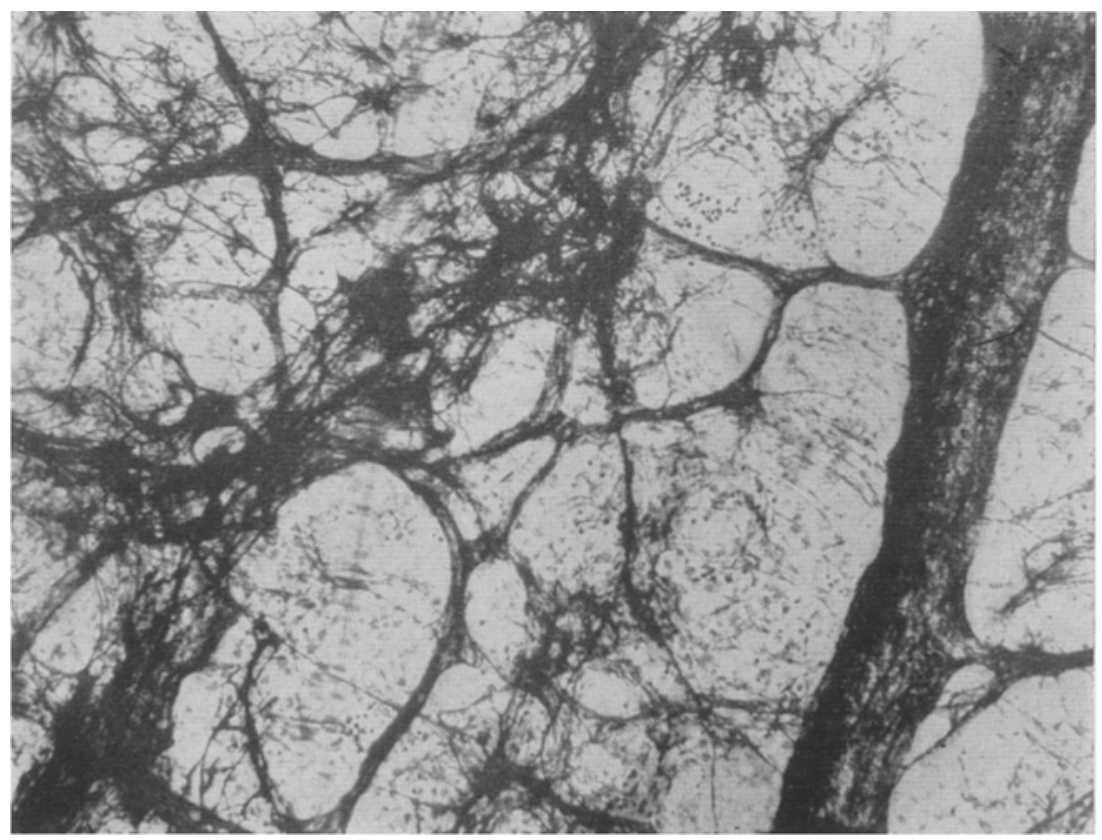

Abb. 16. Mittlere Schicht der Adventitia mit zahlreichen großen, ovalen Zellen mit langen Fortsätzen, welche das Netzwerk der Adventitia und das Bindegewebe der Gefäßmaschen verstärken. - Silberkarbonat, Bindegewebsvariante nach Hortega-Scharenberg; $\times 60$. 


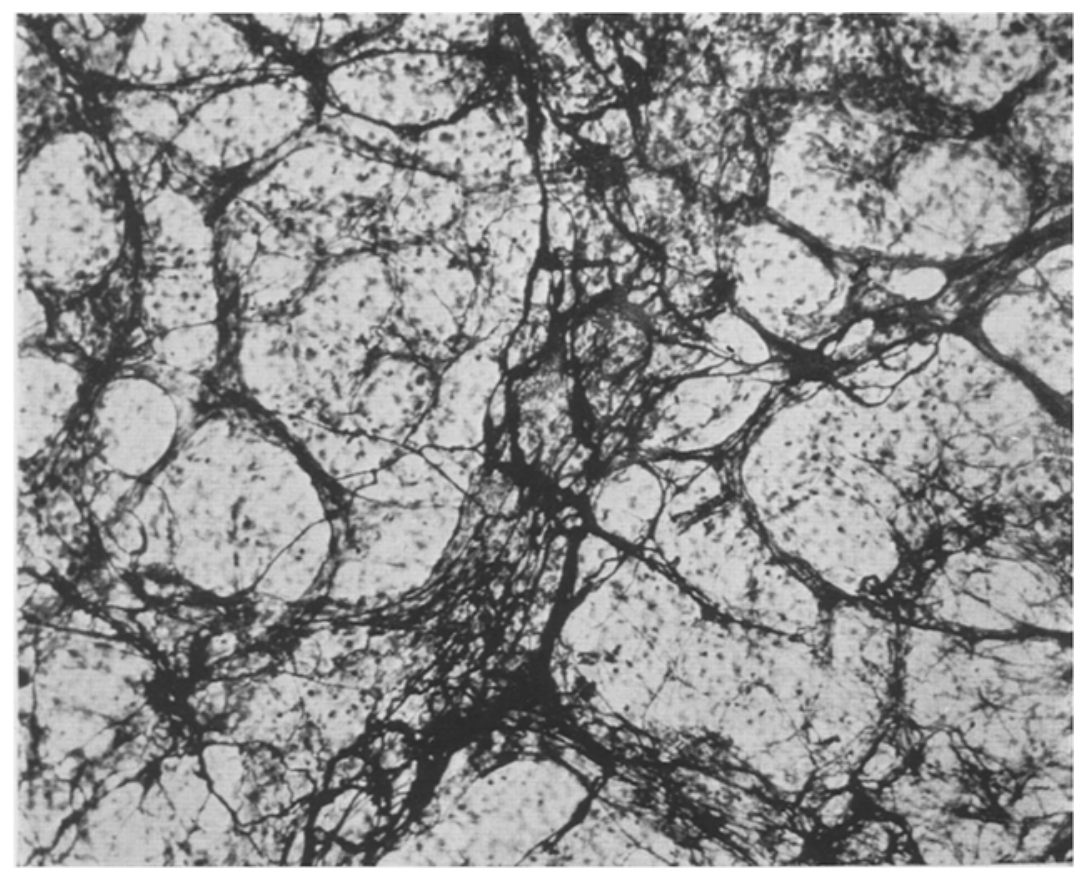

Abb. 17. Tiefere Lagen der Adventitia mit zahlreichen ungleichmäßBigen Verdickungen der Bindegewebsfasern. - Silberkarbonat, Bindegewebsvariante nach Hortega-Scharenberg; $\times 60$,

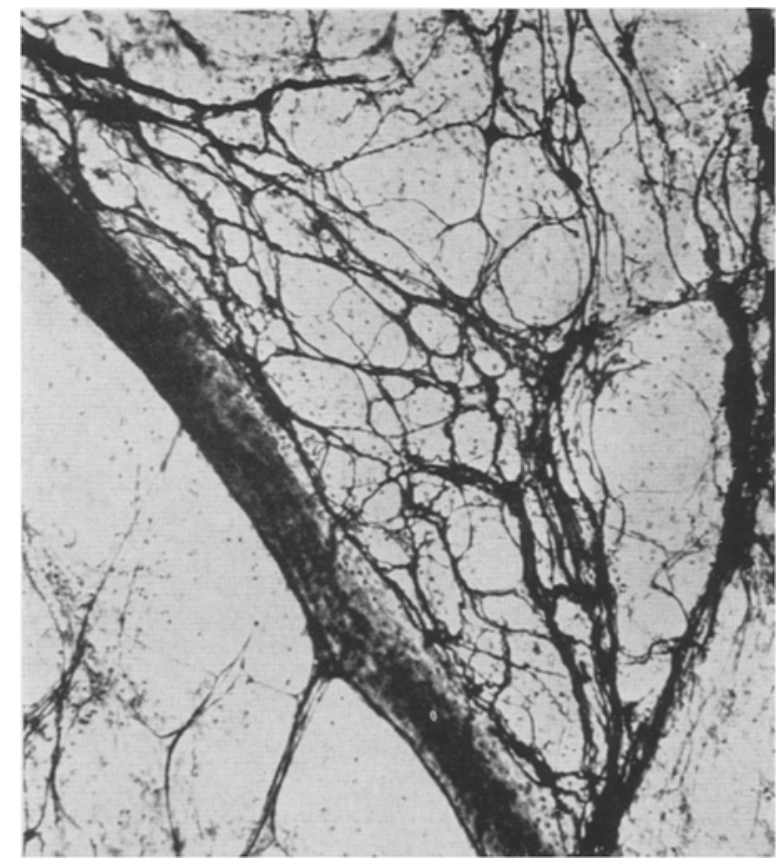

Abb. 18. Fasern der tieferen Schicht der Adventitia. Seitenansicht, welche die Beziehungen zur Media und dem Bindegewebe der Gefäßmaschen wiedergibt. - Silberkarbonat, Bindegewebsvariante nach Hortega-Scharenberg; $\times 60$. 


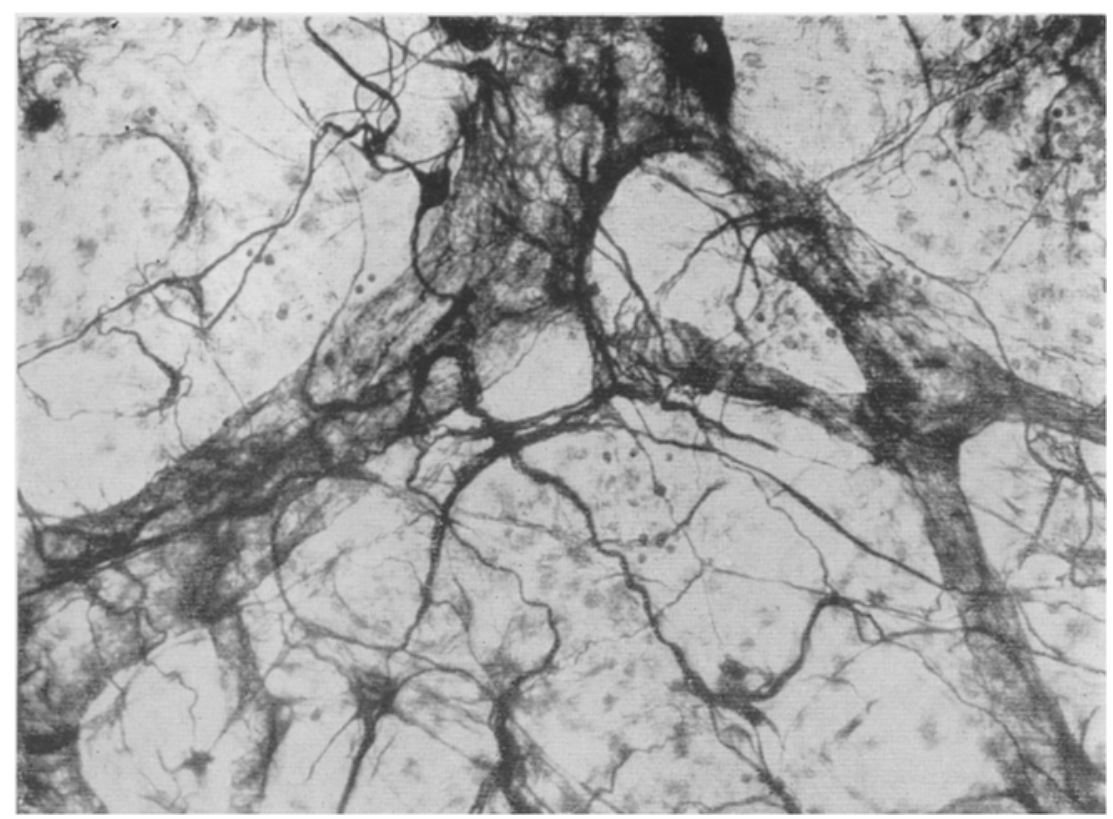

Abb. 19. Ein Teil des Bindegewebes der tieferen Lage der Adventitia mit einem deutlichen birnenförmigen Körper, dessen Fortsätze sowohl mit den Strukturen der Adventitia als auch mit dem Gewebe der Gefäßmaschen in Verbindung stehen. Die der Media anliegenden Bindegewebsfasern bilden ein dichtes aber feines Netz. - Silberkarbonat, Bindegewebsvariante nach Hortega-Scharenberg; $\times 60$.

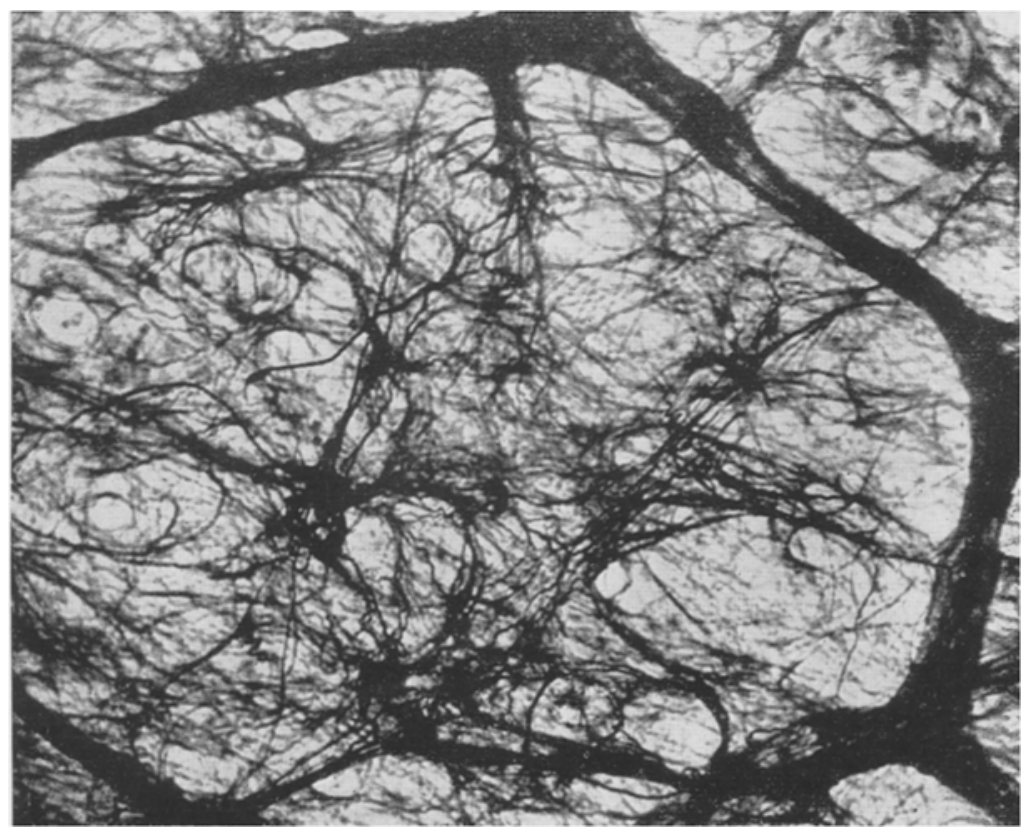

Abb. 20. Sternartig verzweigte Bindegewebsstrukturen, welche die Gefäßmaschen ausfüllen. Silberkarbonat, Bindegewebsvariante nach Hortega-Scharenberg; $\times 60$.

Acta Neurovegetativa, Bd. XVII, Meft $3-4$. 
förmige Zellen, die sehr zahlreiche und lange Ausläufer haben. Ein Teil derselben verstärkt die Adventitia, die anderen bilden einen Teil der Fasern, welche zwischen den Blutgefäßen ausgespannt sind (Abb. 16, 17, 18).

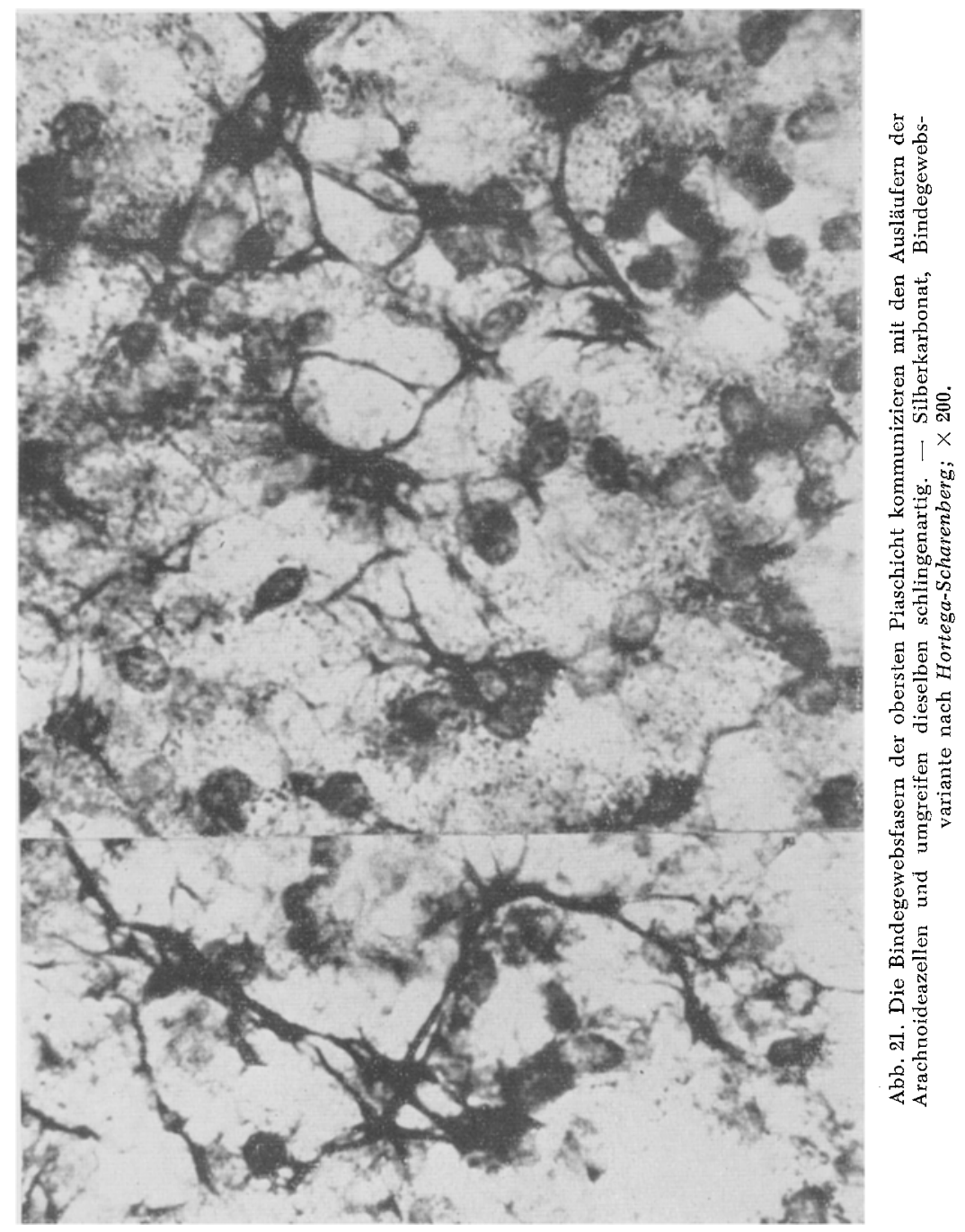

Wenn das Bindegewebe beim Abreißen der Pia teilweise entfernt wird, so lassen sich diese tieferen Schichten der Adventitia besonders gut darstellen. Die 3. Schicht ist durch eigenartige, längliche oder ovale Verdickungen ihrer Fasern ausgezeichnet, die ein relativ grobmaschiges Netz bilden (Abb. 17). Wie eng diese eigenartigen Strukturen der Gefäßwand anliegen, lassen Längsschnitte erkennen (Abb. 18). 
Als 4. Schicht breitet sich ein dichtes Netz feinster Fibrillen unterhalb der Lage der länglichen Fasern (3) und aus demselben hervorgehend aus. Diese 4. Schicht liegt der Media an (Abb. 19). Oft sind in solchen Präparaten einzelne birnenförmige Gebilde enthalten, die sowohl mit der Adventitia als auch mit dem Bindegewebsnetz verbunden sind (Abb. 19).

B. Die Maschen zwischen den Gefäßen sind von charakteristisch gestalteten Bindegewebsstrukturen ausgefüllt. Dieselben bestehen aus sternförmigen, sich überkreuzenden Faserbündeln, die ein dichtes Netz bilden. Dasselbe geht in die Adventitia über (Abb. 20). In den obersten, der Arachnoidea anliegenden Schichten bilden die Ausläufer der sternförmigen Strukturen zarte, mehrschichtige Faserformationen, welche mit den Ausläufern der Arachnoidalzellen kommunizieren und die Elemente schlingenartig umgeben (Abb. 2I).

Es ist offensichtlich, daß die Strukturen des Bindegewebes den Bedürfnissen der Gefäße und der Nerven angepaßt sind. Überall sind die Nerven von Bindegewebsfasem und Zellen umgeben. In der Adventitia lassen sich 4 Schichten unterscheiden: die oberste ist aus dichten, starken, parallel verlaufenden Fibrillen aufgebaut; die 2. enthält große, ovale oder birnenförmige Zellen; die Fasern der 3. Schicht tragen ovale Verdickungen; die 4., der Media anliegende Schicht, besteht aus sehr zarten Fibrillen. Das außerordentlich dichte nervöse Grundnetz dagegen wird von einem entsprechend stark entwickelten, sich überkreuzenden Stützgewebe getragen. Ưberall ist eine besonders zweckmäßige Ordnung erkennbar.

Es besteht eine direkte Verbindung zwischen den Zellen der Arachnoidea und dem Bindegewebe.

\section{Zusammenfassung}

Die Nerven der Pia des Menschen können mit der Silberkarbonattechnik von del Rio Hortega an Ganzpräparaten mikrophotographisch klar wiedergegeben und zwanglos in 2 Systeme eingeteilt werden:

1. Das perivaskuläre System, das von Nerven gebildet wird, die nach ihrem Eintritt in die Pia direkt zu den Gefäßen verlaufen und die letzteren mit einem dichten Geflecht umgeben.

2. Das Grundnetz, welches von Nerven gebildet wird, die a) gleich nach ihrem Eintritt in die Pia sich aufsplittern und den Hauptteil des Grundnetzes bilden; b) von Nerven, welche von einem gefäßwärts verlaufenden Nerven sich abzweigen; c) von Nerven, welche zum perivaskulären Plexus gehören, und schließlich d) von starken Nervenbündeln, welche innerhalb des Grundnetzes zahlreiche Plexus bilden und allmählich in dasselbe übergehen.

Das Grundnetz selbst erscheint histologisch als ein in sich geschlossenes Ganzes und enthält keine besonderen Endformationen.

Die Strukturen des Bindegewebes zerfallen ebenfalls in 2 Systeme: A. das perivaskuläre Gewebe und B. das die Maschen der Gefäße ausfüllende Netz.

A. Die oberflächliche Schicht der Adventitia besteht aus dichten, gleichmäßig starken, parallel verlaufenden Fasern. Die 2. Lage enthält große, ovale oder birnenfönmige Zellen mit zahlreichen Ausläufern, welche in der Literatur vielfach als Ganglienzellen gedeutet worden sind. Die 3. Schicht ist durch ovale Auftreibungen ihrer Fasern charakterisiert und die 4. besteht aus zarten, der Media anliegenden Fibrillen. 
B. Die Maschen zwischen den Gefäßen sind von einem dichten Gewirr sich sternförmig kreuzender Fasern ausgefüllt.

Es bestehen direkte Verbindungen zwischen dem Bindegewebe und den ZelIen der Arachnoidea.

\section{Summary}

The nerves and connective tissue of the human pia were investigated with the silver carbonate method of del Rio Hortega.

The nerves of the pia form two distinctly different but closely associated systems: 1 . the perivascular system is made up of nerves which enter the pia, give numerous branches to the ground network (Grundnetz) (Fig. 1), and form perivascular plexuses (Figs. 2, 3, 4) and, 2. the ground network (Grundnetz), which spreads out over the entire pia and is supported by connective tissue structures. The ground network is formed by: A) nerves which enter the pia and split into numerous branches (Fig. 5), B) ramification of perivascular nerves (Figs. 6, 7, 8, 9), C) numerous plexuses derived from coarse nerves which have no direct connection with the vessels; these plexuses gradually merge with the ground network (Figs. 10, 11, 12). The ground network is a dense interwoven structure without demonstrable terminal formations (Fig. 13). The latter have been found only on the media of vessels (Fig. 14).

The connective tissue structures are no less complicated than those of the nerves and can also be subdivided into two systems: 1 . the adventitia, and 2. the interwoven network of stellate fibers which fills in the space between the vessels.

In the adventitia there can be distinguished four layers: A) the upper which contains coarse parallel fibers (Fig. 15), B) the second which is characterized by large, oval or round elements with numerous processes (Fig. 16), C) the third which is composed of fibers with numerous bead-like swellings along their course (Figs. 17, 18), and D) the fourth which consists of delicate fibers which lie directly on the media (Fig. 19).

The meshes between the vessels are filled with stellate fiber formations (Fig. 20).

The connective tissue fibers of the upper strata of the pia are connected with the processes of the cells of the arachnoidea and are surrounded by numerous connective tissue loops (Fig. 21).

\section{Résumé}

Les nerfs et le tissue conjonctif de la pie-mère humaine furent vérifiés à l'aide de la microphotographie de pièces du tissue entier imprégnées à la méthode de del Rio Hortega. Les nerfs montrent deux systèmes différents:

1. Le système périvasculaire formé par des nerfs qui en entrant dans la piemère joignent les vaisseaux en entournant ceux-ci avec un réseau dense.

2. Le réseau basal contient des nerfs qui a) se ramifient aussitôt entrés dans la pie-mère en formant la plus grande partie du réseau basal; b) des ramifications nerveuses détachées d'un plexus à direction périvasculaire; c) des nerfs qui appartiennent au plexus périvasculaire; d) des gros nerfs qui forment beaucoup des plexus dans le réseau basal pour se fendre graduellement dans celui-ci.

Le réseau basal montre histologiquement une unité sans formations terminales.

Les structures conjonctives se partagent aussi en deux systèmes: A) le tissue périvasculaire et $B$ ) les filets remplissent les mailles des réseaux vasculaires.

A) La couche superficielle de l'adventice est constituée par des fibrilles denses. d'une grosseur égale à cours parallèle. La seconde couche contient des grandes cellules ovales ou piriformes avec beaucoup de prolongements. Ces cellules furent 
souvent décrites comme ganglionnaires. La 3ième couche est caractérisée par des gonflements des fibrilles et la 4ième est constituée de fibrilles minces voisines à la média.

B) Les mailles entre les vaissaux sont remplies d'un réseau dense de fibrilles se croisant à forme étoilée.

Il y a des communications directes entre le tissue conjonctif et les cellules de l'arachnoïde.

\section{Literatur}

1. Stöhr, Ph., jr., Das peripherische Nervensystem. Handbuch der mikrosk. Anatomie des Menschen. Hrsg. von W. v. Moellendorff. Band IV/I. Springer, Berlin, 1928. - 2. Schultz, A., und H. J. Knibbe, Neue Erkenntnisse über die normale und pathologische Histologie der weichen Hirnhäute. Frankf. Zschr. Path. 63 (1952), 472. - 3. Key und Retzius, zit. nach Schaltenbrand und Dorn, Handbuch der mikrosk. Anatomie des Menschen. W. v. Moellendorff und W. Bargmann. Bd. IV/II. Springer, Berlin, 1955. - 4. Scharenberg, $K$., und W. Zeman, Zur Leistungsfähigkeit und zur Technik der Hortega'schen Silberkarbonatmethoden. Arch. Psychiatr. und Zbl. Neurochir. 188 (1952), 430. - 5. Kautzky, R., und J. R. Wolter, Leptomeningiale Äste des Nervus trigeminus. Zbl. Nervenhk. 168 (1952), 24. - 6. Snessareff, $P$., Über die Pigmentzellen Piae Matris beim Menschen. Zschr. wiss. Biol. 9 (1929), 683. - 7. Zit. nach Stöhr, Ph., Acta neuroveget., Wien, 10 (1954), 81 . -

Anschrift des Verfassers: Prof. Dr. K. Scharenberg, University of Michigan Hospital, Ann Arbor, Michigan, USA. 